\title{
ON THE UNIQUENESS OF WEAK SOLUTIONS FOR THE 3D PHASE FIELD NAVIER-STOKES VESICLE-FLUID INTERACTION MODEL
}

\author{
JiHONG ZhaO, QiaO LiU AND SHUANGHU ZHANG
}

\begin{abstract}
In this paper, we study a hydrodynamical system modeling the deformation of vesicle membrane in incompressible viscous fluids. In three dimensional case, we establish some uniqueness criteria of weak solutions for this system which reveal that the regularity of velocity field alone controls the uniqueness of weak solutions.
\end{abstract}

Mathematics subject classification (2010): 35A02, 35G31, 76D09, 76T10.

Keywords and phrases: Navier-Stokes equations, phase field, vesicle-fluid interaction, weak solution, uniqueness.

\section{REFERENCES}

[1] M. AB KARIAN, C. LARTIGUE AND A. Viallat, Tank treading and unbinding of deformable vesicles in shear flow: Determination of the lift force, Phys. Rev. Lett., 88 (2002), 068103.

[2] Q. Chen, C. MiAo AND Z. Zhang, On the uniqueness of weak solutions for the 3D Navier-Stokes equations, Ann. I. H. Poincaré, 26 (2009), 2165-2180.

[3] K. H. De HaAs, C. Blom, D. van den Ende, M. H. G. Duits and J. Mellema, Deformation of giant lipid bilayer vesicles in shear flow, Phys. Rev. E, 56 (1997), 7132-7137.

[4] Q. Du, M. Li AND C. LiU, Analysis of a phase field Navier-Stokes vesicle-fluid interaction model, Discrete Contin. Dyn. Syst. B, 8, 3 (2007), 539-556.

[5] Q. Du, C. LiU, R. RYhAm AND X. WANG, A phase field formulation of the Willmore problem, Nonlinearity, 18 (2005), 1249-1267.

[6] Q. Du, C. LIU, R. RYHAM AND X. WANG, Energetic variational approaches in modeling vesicle and fluid interactions, Physica D, 238 (2009), 923-930.

[7] Q. DU, C. LIU AND X. WANG, A phase field approach in the numerical study of the elastic bending energy for vesicle membranes, J. Computational Physics, 198 (2004), 450-468.

[8] Q. DU, C. LIU AND X. WANG, Retrieving topological information for phase field models, SIAM J. Appl. Math., 65 (2005), 1913-1932.

[9] Q. DU, C. LIU AND X. WANG, Simulating the deformation of vesicle membranes under elastic bending energy in three dimensions, J. Computational Physics, 212 (2006), 757-777.

[10] L. EscauriaZa, G. Seregin AND V. S̆VeráK, $L_{3, \infty}$-solutions to the Navier-Stokes equations and backward uniqueness, Russian Math. Surveys, 58 (2003), 211-250.

[11] W. HelfRICH, Elastic properties of lipid bilayers: theory and possible experiments, Z. Naturforsch., 28, c (1973), 693-703.

[12] I. Gallagher And F. Planchon, On global infinite energy solutions to the Navier-Stokes equations in two dimensions, Arch. Rational Mech. Anal., 161 (2002), 307-337.

[13] P. Germain, Multipliers, paramultipliers, and weak-strong uniqueness for the Navier-Stokes equations, J. Differential Equations, 226 (2006), 373-428.

[14] Y. GIGA, Solutions for semilinear parabolic equations in $L^{p}$ and regularity of weak solutions of the Navier-Stokes system, J. Differential Equations, 61 (1986), 186-212.

[15] H. Kozono AND H. SoHR, Remark on uniqueness of weak solutions to the Navier-Stokes equations, Analysis, 16 (1996), 255-271.

[16] H. Kozono AND Y. TANIUCHI, Bilinear estimates in BMO and the Navier-Stokes equations, Math. Z., 235 (2000), 173-194. 
[17] P.-G. Lemarié-Rieusset, Recent Developments in the Navier-Stokes Problem, Research Notes in Mathematics, Chapman \& Hall/CRC, 2002.

[18] Y. LiU, T. TAKAhAShi AND M. TUCSNAK, Strong solution for a phase field Navier-Stokes vesicle fluid interaction model, J. Math. Fluid Mech., 14 (2011), 177-195.

[19] L. Miao, U. SEIfERT, M. Wortis ANd H.-G DöBEREINER, Budding transitions of fluid-bilayer vesicle: The effect of area-difference elasticity, Phys. Rev. E, 49 (1994), 5389-5407.

[20] O. Mouritsen, Life-As a Matter of Fat: The Emerging Science of Lipidomics, Springer, Berlin, 2005.

[21] Z. OU-YANG AND W. HelfRICH, Bending energy of vesicle membranes: General expressions for the first, second and third variation of the shape energy and applications to spheres and cylinders, Phys. Rev. A, 39 (1989), 5280-5288.

[22] G. Prodi, Un teorema di unicità per le equazioni di Navier-Stokes, Ann. Math. Pura Appl., 48, 1 (1959), 173-182.

[23] F. RIBAUD, A remark on the uniqueness problem for the weak solutions of Navier-Stokes equations, Ann. Fac. Sci. Toulouse Math., 11 (2002), 225-238.

[24] U. Seifert And R. Lipowsky, Morphology of Vesicles, in: Handbook of Biological Physics, Volume 1, Elsevier Science B.V, 1995.

[25] J. SERRIN, The initial value problem for the Navier-Stokes equations, in: R.E. Langer (Ed.), Nonlinear Problems, University of Wisconsin Press, Madison, (1963), 69-98.

[26] W. VON WAHL, The equations of Navier-Stokes and abstract parabolic equations, Vieweg and Sohn, Wiesbaden, 1985.

[27] H. WU AND X. XU, Strong solutions, global regularity and stability of a hydrodynamic system modeling vesicle and fluid interactions, SIAM J. Math. Anal., 45, 1 (2013), 181-214.

[28] J. ZHAO, A Beale-Kato-Majda blow-up criterion for a hydrodynamic system modeling vesicle and fluid interactions, arXiv:1208.3800v1. 